\title{
Controlled Invariant Polyhedral Sets for Constrained Discrete-Time Descriptor Systems
}

\author{
José Mario Araújo ${ }^{1}$ and Carlos Eduardo Trabuco Dórea ${ }^{2}$ \\ ${ }^{1}$ Instituto Federal da Bahia, Departamento de Tecnologia em Eletro-Eletrônica, Rua Emídio \\ dos Santos, S/N, Barbalho, Salvador-BA, Brazil \\ jomario@ifba.edu.br \\ ${ }^{2}$ Universidade Federal da Bahia, Escola Politécnica, Depto. de Engenharia Elétrica, \\ Rua Aristides Novis, 2, Federação, Salvador-BA, Brazil \\ cetdorea@ufba.br
}

\begin{abstract}
This paper addresses the problem of constructing controlled invariant polyhedral sets for linear discrete-time descriptor systems subject to state and control constraints and persistent disturbances. Regardless the large number of contributions on set invariance for linear systems in the standard form, there are few works dealing with set invariance properties in the case of descriptor systems. Here, assuming regularity and causality of the descriptor system, the state equations are written in such way that standard algorithms can be directly applied. Moreover, state and control constraints can be enforced through a piecewise linear delayed state feedback. A numerical example is presented to illustrate these ideas.
\end{abstract}

Keywords: Descriptor systems, set invariance, constraints.

\section{Introduction}

Descriptor systems is an important class of linear systems, also called generalized state space, or singular systems and yet implicit systems when algebraic equations are present. Very important works have built a rich theoretical and applied framework on the subject [1], [2], [3], [4] and [5]. The design of constrained control systems is very useful in practice, since certain requirements of supply or safety impose limits on physical quantities. The use of the set-invariance concept is a efficient way of dealing with constrained control systems (see [6], [7] and [8] and references therein). Several works concerning constrained control for standard state-space systems can be found in the literature [9], [10] and [11]. For the class of descriptor linear systems, however, the contributions on the characterization of controlled invariance over polyhedral sets are not so numerous. Some important works on positive invariance under linear pole placement design can be found on literature [12], [13], [14], [15] and [16]. However, the use of linear feedback may lead to very conservative solutions, not coping with severe control constraints or high amplitude disturbances. In this note, controlled 
invariance of polyhedral sets for discrete time linear descriptor systems is analyzed. By assuming that the system is regular and causal, the state equation can be rewritten in an augmented form which gives the possibility of applying available methods for characterization of controlled invariance for polyhedral sets in standard linear systems. A delayed state-feedback control law can then be synthesized to enforce the constraints. A numerical example is presented in order to illustrate the effectiveness of the proposal.

\section{Contribution for Technological Innovation}

Descriptor systems arise from a diversity of areas, such economics, chemical and electrical [1]. The control of this class of models is very important, since a series of requirements of quality, reliability and safety are involved. In many cases, physical constraints are inherent, and comprising operational limits of supply/safety. The setinvariance for control under constraints is well developed for systems in the classical state representation. For descriptor model a few works have contributed in this aspect. The present paper tries to rise new ideas in this direction.

\section{Preliminaries}

The nth-dimensional system under study is in the form of a perturbed descriptor statespace representation:

$$
E x(k+1)=A x(k)+B u(k)+D d(k)
$$

in which $x \in \mathfrak{R}^{n}, u \in \mathfrak{R}^{m}, d \in \mathfrak{R}^{p}$. The disturbance amplitude is supposed to be bounded for all instants $k$. When $\operatorname{rank}(E)=q<n$, without loss of generality, the state vector can be partitioned:

$$
x(k+1)=\left[\begin{array}{c}
x_{1}(k+1) \\
x_{2}(k+1)
\end{array}\right]
$$

and the system matrices can be written:

$$
E=\left[\begin{array}{cc}
I_{q} & 0 \\
0 & 0
\end{array}\right], A=\left[\begin{array}{ll}
A_{11} & A_{12} \\
A_{21} & A_{22}
\end{array}\right], B=\left[\begin{array}{l}
B_{1} \\
B_{2}
\end{array}\right], D=\left[\begin{array}{c}
D_{1} \\
D_{2}
\end{array}\right]
$$

The hypothesis that the system is causal assures that $A_{22}$ is invertible [4]. In this case, manipulations on the systems matrices leads to the form:

$$
x(k+1)=\tilde{A} x(k)+\tilde{B} u(k)+\tilde{C} u(k+1)+\tilde{D}\left[\begin{array}{c}
d(k) \\
d(k+1)
\end{array}\right]
$$


in which

$$
\begin{gathered}
\tilde{A}=\left[\begin{array}{cc}
A_{11}-A_{12} A_{22}^{-1} A_{21} & 0 \\
-A_{22}^{-1} A_{21}\left(A_{11}-A_{12} A_{22}^{-1} A_{21}\right) & 0
\end{array}\right], \tilde{B}=\left[\begin{array}{c}
B_{1}-A_{12} A_{22}^{-1} B_{2} \\
-A_{22}^{-1} A_{21}\left(B_{1}-A_{12} A_{22}^{-1} B_{2}\right)
\end{array}\right] \\
\tilde{C}=\left[\begin{array}{c}
0 \\
-A_{22}^{-1} B_{2}
\end{array}\right], \tilde{D}=\left[\begin{array}{cc}
D_{1}-A_{12} A_{22}^{-1} D_{2} & 0 \\
-A_{22}^{-1} A_{21}\left(D_{1}-A_{12} A_{22}^{-1}\right) & -A_{22}^{-1} D_{2}
\end{array}\right]
\end{gathered}
$$

Now, one can see that one-step forward terms in control input and in disturbance appear. This is not a problem for the disturbance, since it is not measured. For the control input, it is convenient to consider it as another state variable. The augmented state vector is then:

$$
\chi(k+1)=\left[\begin{array}{c}
x(k+1) \\
u(k+1)
\end{array}\right]
$$

The augmented state equation are now given as:

$$
\chi(k+1)=\left[\begin{array}{cc}
\tilde{A} & \tilde{B}+\tilde{C} \\
0 & I
\end{array}\right] \chi(k)+\left[\begin{array}{c}
\tilde{C} \\
I
\end{array}\right] \Delta u(k+1)+\left[\begin{array}{c}
\tilde{D} \\
0
\end{array}\right]\left[\begin{array}{c}
d(k) \\
d(k+1)
\end{array}\right]
$$

In which $\Delta u(k+1)=u(k+1)-u(k)$. This is a standard state-space model with state vector $\left[\begin{array}{lll}x^{T}(k) & u^{T}(k)\end{array}\right]^{T}$ and bounded disturbance $\left[\begin{array}{lll}d^{T}(k) & d^{T}(k+1)\end{array}\right]^{T}$, and application of well-established algorithms for standard systems is straightforward. It should be noticed that this extended system has $n+m$ eigenvalues: (i) $q$ finite eigenvalues of (1); (ii) $n-q$ eigenvalues equal to 0 corresponding to infinite eigenvalues of (1); and $m$ eigenvalues equal to 1 corresponding to the control input dynamics. In the next section, this representation will be useful for the characterization of controlled invariance of polyhedral sets that represent constraints on state variables and control inputs.

\section{Solution of Constrained Control Problem}

\subsection{Controlled Invariance}

Assume that system (1) is subject to the following state and control constraints:

$$
x(k) \in \Omega=\{x: G x \leq \rho\}, u(k) \in \mathcal{U}=\{u: U u \leq v\}, \Delta u(k) \in \Delta \mathcal{V}=\{\Delta u: L \Delta u \leq \varphi\}
$$

The disturbance is supposed to be bounded as follows:

$$
d(k) \in \mathcal{D}=\{d: V d \leq \mu\}
$$


Considering the augmented representation, the constraints on state and control can be written as:

$$
\chi(k) \in \Gamma=\{\chi: \mathcal{G} \chi \leq \rho\}, \mathcal{G}=\left[\begin{array}{cc}
G & 0 \\
0 & U
\end{array}\right], \rho=\left[\begin{array}{l}
\rho \\
v
\end{array}\right]
$$

and the bounds on the disturbance as:

$$
\left[\begin{array}{c}
d(k) \\
d(k+1)
\end{array}\right] \in \Psi=\{d: \mathcal{V} d \leq \bar{\mu}\}, \mathcal{V}=\left[\begin{array}{cc}
V & 0 \\
0 & V
\end{array}\right], \bar{\mu}=\left[\begin{array}{c}
\mu \\
\mu
\end{array}\right]
$$

The goal now is to construct a control law which enforces the constraints, for all disturbances in $\Psi$. Such a construction will be based on the following concept [9]:

Definition 4.1. A set is $\lambda$-contractive controlled invariant with respect to system (6) and constraints (7),(8) if for any $\chi(k) \in \Gamma, \exists \Delta u$ such that

$$
\chi(k+1)=\left[\begin{array}{cc}
\tilde{A} & \tilde{B}+\tilde{C} \\
0 & I
\end{array}\right] \chi(k)+\left[\begin{array}{c}
\tilde{C} \\
I
\end{array}\right] \Delta u(k+1)+\left[\begin{array}{c}
\tilde{D} \\
0
\end{array}\right]\left[\begin{array}{c}
d(k) \\
d(k+1)
\end{array}\right] \in \lambda \Gamma \quad \text { with } 0<\lambda \leq 1
$$

The constrained control problem can then be solved by computing $\Gamma^{*}$, the maximal controlled invariant set contained in $\Gamma[8,9]$. Hence, if the initial state is consistent with the algebraic equation:

$$
0=A_{21} x_{1}(0)+A_{22} x_{2}(0)+B_{2} u(0)+D_{2} d(0)
$$

and $\chi(k) \in \Gamma^{*}$ then there exists $\Delta u(k) \in \Delta \mathcal{V}$ such that $\chi(k+1) \in \lambda \Gamma^{*}, \forall d(k) \in \mathcal{D}$. As a consequence, if $\chi(0) \in \Gamma^{*}$, then $\exists \Delta u(k+1)$ such that $\chi(k) \in \Gamma^{*} \forall k$ and $\forall d(k) \in \mathcal{D}$. Moreover, if $d(k)=0, \chi(k) \in \lambda^{k} \Gamma^{* k}$, thus if $\lambda<1, \chi(k) \rightarrow 0$ when $k \rightarrow \infty$, guaranteeing asymptotic stability.

Once the maximal controlled invariant is obtained, a piecewise linear control law can be computed that assures the respect to constraints [9]:

$$
\Delta u(k+1)=\phi[\chi(k)]=\phi[x(k), u(k)]
$$

One can see that such control law is delayed with respect to the augmented state vector.

\subsection{Admissible Initial Conditions}

In the previous subsection, the issue of consistency of initial conditions was mentioned. The control law (10) enforces state and control constraints as long as the initial state satisfies $\chi(0) \in \Gamma^{*}$. However, differently from standard linear systems, $\chi(0)$ may not be consistent, i.e., may not satisfy the algebraic equation (9). In this 
case, a jump can occur for $k=0$ and this is unpredictable due the disturbance $d(0)$. Let $x\left(0^{+}\right)$be the state after the jump. The dynamic part of state vector does not experiment jumps in $k=0$. It can be verified by changing the representation of (1) into a canonical representation where the dynamic and algebraic equations are decoupled [14]. Hence $x_{1}\left(0^{+}\right)=x_{1}(0)$. The algebraic portion can be made explicit from (9):

$$
x_{2}\left(0^{+}\right)=-A_{22}^{-1} A_{21} x_{1}(0)-A_{22}^{-1} B_{2} u(0)-A_{22}^{-1} D_{2} d(0)
$$

The consistency of the initial condition can be treated on the basis of the following definition.

Definition 4.2. Consider the set $\Gamma^{*}$, controlled invariant with respect to the system (6) and constraints (7),(8). The set of admissible initial states is defined by: $\Lambda_{a d}=\left\{x(0) \in \Omega: \exists u(0) \in \mathcal{U}: x\left(0^{+}\right) \in \Gamma^{*}, \forall d(0) \in \mathcal{D}\right\}$.

Let now $\Gamma^{*}$ be the polyhedral set $\Gamma^{*}=\left\{x: G^{*} x \leq \rho^{*}\right\}$. In this case, the set of admissible initial states is also a polyhedron, given by the set of $x(0)=\left[x_{1}(0)^{T} \quad x_{2}(0)^{T}\right]^{T}$ such that $\exists u(0)$ which satisfies, $\forall V d(0) \leq \mu$ :

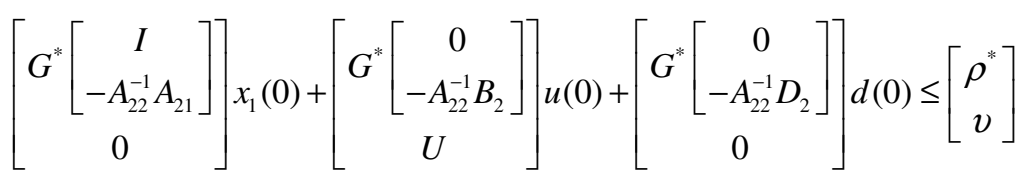

Since $d(0)$ is not measured, its influence can be taken into account by considering the worst case row-by-row. Define then the vector:

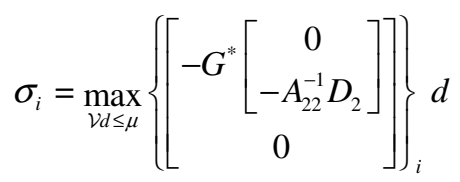

The variable $u(0)$ can be eliminated by a projection computed like in [8], resulting in the following polyhedron on $x_{1}$ :

$$
\Lambda_{x_{1}}=\left\{x_{1}(0): H_{1} x_{1}(0) \leq h_{1}\right\}
$$

The set of admissible initial states is then given by:

$$
\Lambda_{a d}=\left\{x(0):\left[\begin{array}{cc}
{\left[\begin{array}{ll}
H_{1} & 0
\end{array}\right]} \\
G
\end{array}\right] x(0) \leq\left[\begin{array}{c}
h_{1} \\
\rho
\end{array}\right]\right\}
$$



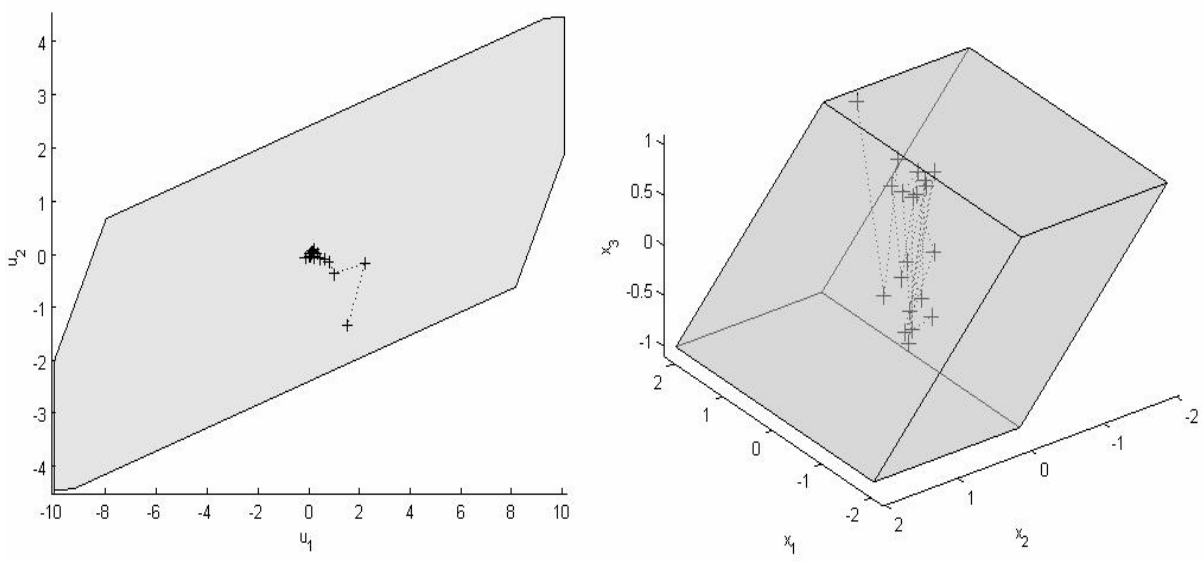

Fig. 1. Projection onto the control space (a) and the state space (b) of the controlled invariant polyhedron computed with $\gamma=1$, together with a control and a state trajectory

\section{Numerical Example}

Consider the same system used in $[13,14,15]$ :

$$
E=\left[\begin{array}{lll}
1 & 0 & 0 \\
0 & 1 & 0 \\
0 & 0 & 0
\end{array}\right], A=\left[\begin{array}{ccc}
1.2 & 0 & 0 \\
-1 & -0.7 & -1 \\
2 & -0.5 & -1.2
\end{array}\right], B=\left[\begin{array}{cc}
0 & 1 \\
1 & -1 \\
0.5 & 2
\end{array}\right], D=\left[\begin{array}{l}
0 \\
1 \\
1
\end{array}\right]
$$

The following state and control constraints (7),(8) are imposed: $\left|G_{s} x\right| \leq \rho_{s},\left|u_{i}\right| \leq 10, i=1,2$ with

$$
G_{s}=\left[\begin{array}{ccc}
0 & 1 & 0.9318 \\
-1 & 0 & -0.1164 \\
0 & 0 & 1
\end{array}\right], \rho_{s}=\left[\begin{array}{l}
1 \\
2 \\
1
\end{array}\right]
$$

The disturbances are bounded as $|d(k)| \leq \gamma$. By application of the algorithm proposed in [8], it was verified that the maximal controlled invariant polyhedron contained in the set of constraints, with a contraction rate $\lambda=0.99$, is non-empty for $\gamma \leq \gamma_{\max }=1.082115$.

Figure 1 shows the controlled invariant polyhedron for $\gamma=1$ projected onto control and state spaces, where it can be noticed that the original polyhedron of the constraints in states is itself controlled invariant. These results are better than those obtained by the pole placement control law proposed in [14], where $\gamma_{\max }=0.208815$. A piecewise control law that enforces the constraints can be computed as in [9]. However, it turns out that for this example, with $\gamma=1$, invariance of $\Omega$ under control 
constraints can be achieved by a linear feedback $\Delta u(k+1)=F \chi(k)=F_{1} x(k)+F_{2} u(k)$ computed by linear programming as in $[10,11]$, leading to

$$
F=\left[\begin{array}{ccccc}
0.9370 & 0.7409 & 0 & -0.8944 & -0.4852 \\
-0.7146 & 0.1915 & 0 & -0.0385 & -1.8254
\end{array}\right]
$$

It should be noticed that due to the null column in $F$, the closed-loop augmented systems has: (i) 4 finite eigenvalues in $0.6663,-0.4669,-0.0329,0.0305$, and (ii) 1 eigenvalue equal to 0 , corresponding to the infinite pole of (1). Its confirms stability of the system under closed-loop.

The set of admissible initial states coincides with the initial polyhedron. A simulation was carried out with initial conditions $x(0)=\left[\begin{array}{lll}1.649 & -0.2354 & 0\end{array}\right]^{T}$, $x\left(0^{+}\right)=\left[\begin{array}{lll}1.649 & -0.2354 & 1\end{array}\right]^{T}$. The initial control input $u(0)$ was then computed in order to guarantee $x\left(0^{+}\right) \in \Gamma^{*}$, resulting in $u(0)=\left[\begin{array}{ll}1.5 & -1.344\end{array}\right]^{T}$ The simulated trajectories with random disturbances are depicted in figure 1.

\section{Conclusions and Future Works}

A characterization of controlled invariance of polyhedral sets was proposed for descriptor linear discrete time systems, based on an augmented state-space representation in the form of a classical linear system. Under this representation, standard algorithms can be used to compute controlled invariant sets. Then, a delayed state feedback law can be synthesized to solve a control problem under state and control constraints for systems subject to bounded disturbances. Through a numerical example, it was shown that the proposed controller can outperform linear ones proposed in literature. Future works will focus the study of the solution to the problem by means of static and dynamic output feedback laws, already characterized in [17] for classical linear systems. The design of a control law for simultaneous regularization and constraints satisfaction will be also investigated.

Acknowledgments. The Authors would like to thank to their Institutions and to PROAP/CAPES for financial support.

\section{References}

1. Luenberger, D.G.: Time-invariant descriptor systems. Automatica 14(5), 473-480 (1978)

2. Yip, E.L., Sincovec, R.F.: Solvability, controllability, and observability of continuous descriptor systems. IEEE T Automat. Contr. 26(3), 702-707 (1981)

3. Cobb, D.: Feedback and pole placement in descriptor variable systems. Int. J. Control 33(6), 1135-1146 (1981)

4. Dai, L.: Singular control systems. Springer, New York (1989)

5. Lewis, F.: A survey of linear singular systems. Circ. Syst. Signal Pr. 5(1), 5-36 (1986)

6. Blanchini, F.: Set invariance in control. Automatica 35(11), 1747-1767 (1999) 
7. Dórea, C.E.T., Hennet, J.C.: (A,B)-invariance conditions of polyhedral domains for continuous-time systems. European Journal of Control 5(1), 70-81 (1999)

8. Dorea, C.E.T., Hennet, J.C.: (A,B)-invariant polyhedral sets of linear discrete-time systems. J. Optimiz. Theroy App. 103(3), 521-542 (1999)

9. Blanchini, F.: Ultimate boundedness control for uncertain discrete-time systemsvia setinduced Lyapunov functions. IEEE T Automat. Contr. 39(2), 428-433 (1994)

10. Vassilaki, M., Hennet, J.C., Bitsoris, G.: Feedback control in linear discrete-time systems under state and control constraints. Int. J. Control 47(6), 1727-1735 (1988)

11. Vassilaki, M., Bitsoris, G.: Constrained regulation of linear continuous-time dynamical systems. Syst. Control Lett. 13, 247-252 (1989)

12. Georgiou, G., Krikelis, N.J.: A design approach for constrained regulation in discrete singular systems. Syst. Control Lett., 297-304 (1991)

13. Tarbouriech, S., Castelan, E.B.: Positively invariant-sets for singular discrete-time systems. International Journal of Systems Science 24(9), 1687-1705 (1993)

14. Castelan, E.B., Tarbouriech, S.: Simple and weak D-invariant polyhedral sets for discretetime singular systems. Controle e Automacao 14(4), 339-347 (2003)

15. Tarbouriech, S., Castelan, E.B.: An eigenstructure assignment approach for constrained linear continuous-time systems. S yst. Control Lett. 24, 333-343 (1995)

16. Lin, Z., Lv, L.: Set invariance conditions for singular linear systems subject to actuator saturation. IEEE T Automat. Contr. 52(12), 2351-2355 (2007)

17. Dórea, C.E.T.: Output-feedback controlled invariant polyhedra for constrained linear systems. To appear in Proceedings of 48th CDC, pp. 1-6 (2009) 